\title{
Analysis of causes and clinical pattern of infertility in couples coming to a tertiary care centre in Bihar, India
}

\author{
Kalpana Singh $^{1 *}$, Rekha Kumari ${ }^{1}$, Alok Ranjan², Geetam Bharti ${ }^{1}$
}

\begin{abstract}
${ }^{1}$ Department of Reproductive Biology, Department of Biochemistry, IGIMS, Patna, Bihar, India
${ }^{2}$ Department of Community and Family Medicine, AIIMS, Patna, Bihar, India
\end{abstract}

Received: 26 April 2017

Accepted: 08 May 2017

*Correspondence:
Dr. Kalpana Singh,
E-mail: drkalpana1 @ yahoo.com

Copyright: () the author(s), publisher and licensee Medip Academy. This is an open-access article distributed under the terms of the Creative Commons Attribution Non-Commercial License, which permits unrestricted non-commercial use, distribution, and reproduction in any medium, provided the original work is properly cited.

\section{ABSTRACT}

Background: Infertility is one of the major health problems and a socially destabilizing condition for couples often causing marital disharmony. Infertility is defined as the failure to achieve a clinical pregnancy after 12 months or more of regular unprotected sexual intercourse. Worldwide, its prevalence is approximately 8-12\%.There are numerous factors such as anatomical, physiological and genetic factors that cause infertility. Many environmental and acquired factors also lead to inability to conceive. Objective of the present study was to determine the causes and clinical pattern of infertility in infertile couples.

Methods: It was a hospital based observational study carried out on 750 women in reproductive age group attending out-patient clinic of Reproductive Biology Department of Indira Gandhi Institute of Medical Sciences (IGIMS) at Patna during April 2013 to March 2017. All the cases of primary and secondary infertility diagnosed after full examinations and laboratory tests were included and cases lacking of full examinations and laboratory tests were excluded. All the data of infertile couples were recorded in a semi-structured Case Information Performa.

Results: Out of 750 women 454 women had all the data and they participated in the study Nearly $68 \%$ women had primary infertility and rest had secondary infertility. Male factor was responsible in $37.39 \%$, female factor in $20.48 \%$, unexplained in $22.46 \%$ while a combination of both factor was seen in $8.37 \%$ cases in our study .135 women had irregular menstrual cycles in which $64(47 \%)$ had oligomenohhrea. $79 \%$ women had normal ultrasonography and nearly $11 \%$ of women had evidence of PCOD. $24 \%$ women had hypothyroidism (TSH more than 4.5 IU/L) and $59(13 \%)$ were found to have high level of prolactin i.e. $>25 \mathrm{ng} / \mathrm{ml}$. Nearly $8 \%$ of women had high level of FSH i.e. more than $10 \mathrm{IU} / \mathrm{L}$ which is an indicator of ovarian resistance. In nearly $16 \%$ women one fallopian tube was found blocked and $8 \%$ had both tubes blocked in hysterosalpingography. Husband semen analysis was done to assess male factor. Nearly $14 \%$ of their male partners suffered from azoospermia and $23 \%$ had at least one abnormal parameter in semen analysis.

Conclusions: Etiological pattern of infertility varies in different parts of World. Male and female factors both are responsible for infertility. So, both the partners should be counselled and investigated properly.

Keywords: Cause, Female factor, Infertility, Male factor, Pattern

\section{INTRODUCTION}

Infertility is one of the major health problems and a socially destabilizing condition for couples often causing marital disharmony. Infertility is defined as the failure to achieve a clinical pregnancy after 12 months or more of regular unprotected sexual intercourse. ${ }^{1}$ Worldwide, its prevalence is approximately $8-12 \% .^{2}$ Infertility affects a relatively large number of couples at some point in their 
reproductive lives - globally, between 50 and 80 million couples (WHO, 1994). ${ }^{3}$

The WHO estimates the overall prevalence of primary infertility in India to be between 3.9 and 16.8 per cent. ${ }^{4}$ Estimates of infertility vary widely among Indian states from $3.7 \%$ in Uttar Pradesh, Himachal Pradesh and Maharashtra, to 5 per cent in Andhra Pradesh, and 15 per cent in Kashmir. ${ }^{5-7}$ Moreover, the prevalence of primary infertility has also been shown to vary across tribes and castes within the same region in India. ${ }^{5,8}$ However, it should be noted that many of these estimates use different definitions of infertility and consider different time periods, which makes direct comparisons difficult between any studies.

In India, the extent of overall primary and secondary infertility among the women at the end of their reproductive careers (aged 45-49) was reported to be around $8 \% .{ }^{9}$ However; the rate of infertility has decreased by $7.7 \%$ from NFHS-2 to NFHS-3 in India. It is around $2 \%$ in NFHS-2 and around $1.85 \%$ in NFHS-3. ${ }^{10}$ A study conducted among the women in age group $15-30$ years in Mysore reported 12.6 per cent $(95 \%$ Confidence Interval (CI): $10.5-15.0 \%)$ prevalence of primary infertility. ${ }^{11}$

The extent and cause of infertility depends on various socio-cultural factors that directly or indirectly influence sexual practices and behaviours leading to infertility. Various factors like age at marriage, place of residence, social class and educational status of women could be some of the factors of infertility among women in India. Also, there are numerous factors such as anatomical, physiological and genetic factors that cause infertility. Many environmental and acquired factors also lead to inability to conceive. The exact incidence of infertility is difficult to estimate as many eligible couples, especially living in rural and remote areas, do not seek help or consult specialized health care providers in India.

There is paucity of information about the prevalence of primary and secondary infertility among infertile women of reproductive age group in Bihar. Hence a study was planned to assess causes and clinical pattern of infertility among women visiting a tertiary care centre in Patna, Bihar.

\section{METHODS}

It was a hospital based observational study carried out among the women attending out-patient clinic of Reproductive Biology Department of Indira Gandhi Institute of Medical Sciences (IGIMS) at Patna during April 2013 to March 2017.

A total of 750 women in reproductive age group, who attended the clinic for consultation, were included satisfying the inclusion criteria and after obtaining their verbal consent.

\section{Inclusion criteria}

All the cases of primary and secondary infertility diagnosed by the gynaecologist after full examinations and laboratory tests.

\section{Exclusion criteria}

Lack of full examinations and laboratory tests.

\section{Data collection}

A semi-structured Case Information Proforma was developed to capture all relevant information of the eligible couples. Demographic characteristics such as current age, age at the time of marriage, menstrual history, and history of galactorrhoea, sexual desire and sexual activity were obtained through face-to-face interview by the attending physician. The subjects were also clinically examined. Biological specimens of all female patients were collected for the hormonal evaluation such as TSH, T4, T3, Prolactin, Day 2 FSH and LH. These tests were done by chemiluminescent immunoassay method on Access 2 using reagents and calibrators from Backman Coulter and quality control material from Bio-rad Company. Ultrasonography of pelvic organs and hysterosalpingography were performed routinely for tubal evaluation. Seminal fluids analysis was done of all male partners of the infertile couple after an abstinence of 2-5 days and analysed according to World Health Organization 2010 guidelines.

\section{Statistical analysis}

All data from the case information sheet were transferred to the computer using MS Excel sheet (MS Office 7). All statistical analyses were performed using statistical software Stata version 7 (Stata Corp, Texas, and USA).

\section{RESULTS}

A total of 454 women out of 750 participated and gave consent for sharing all relevant information. Records with missing variables were not considered for the analysis.

Table 1: Demographic characteristics of women.

\begin{tabular}{|ll|}
\hline Variables & $\mathbf{N}=\mathbf{4 5 4}(\%)$ \\
\hline Age (years) & \\
\hline$<20$ & $22(4.85)$ \\
\hline $21-30$ & $312(68.72)$ \\
\hline $31-40$ & $106(23.35)$ \\
\hline$>40$ & $14(3.08)$ \\
\hline Duration of marriage (years) & \\
\hline$<2$ & $73(16.08)$ \\
\hline $2-7$ & $230(50.66)$ \\
\hline $8-12$ & $95(20.93)$ \\
\hline $13-17$ & $29(6.39)$ \\
\hline$>17$ & $27(5.95)$ \\
\hline
\end{tabular}


Table 1 presents the demographic characteristics of women. Maximum women $(68.7 \%)$ were in age group 21-30 years, followed by $23.3 \%$ in age group $31-40$ years. The mean age of women at presentation was 28.24 years (95\% CI: 27.7-28.7). The median duration of marriage was 5 years (IQR: 3 to 9 years) in the study population.

Table 2: Clinical characteristics of women.

\begin{tabular}{|c|c|}
\hline Variables & $\mathbf{N}(\%)$ \\
\hline \multicolumn{2}{|l|}{ Parity } \\
\hline 0 & $306(67.55)$ \\
\hline 1 & $85(18.76)$ \\
\hline 2 & $62(13.69)$ \\
\hline \multicolumn{2}{|l|}{ Menstrual history } \\
\hline Regular & $319(70.26)$ \\
\hline Oligomenorrhea & $64(14.10)$ \\
\hline Hypermonorrhoea & $37(8.15)$ \\
\hline Menometrorrhagia & $6(1.32)$ \\
\hline Irregular Menstrual cucle & $3(0.66)$ \\
\hline Menorrhagia & $15(3.30)$ \\
\hline Dysmenorrhoea & $5(1.10)$ \\
\hline Polymenorrhoea & $5(1.10)$ \\
\hline \multicolumn{2}{|l|}{ Ultrasonography } \\
\hline Normal & $361(79.52)$ \\
\hline PCOD & $50(11.01)$ \\
\hline Fibroid & $14(3.08)$ \\
\hline Endometriosis & $4(0.88)$ \\
\hline Endometriosis + Fibroid & $2(0.44)$ \\
\hline Ovarian Cyst & $10(2.20)$ \\
\hline Hydrosalpinx & $4(0.88$ \\
\hline Anatomical defect & $4(0.88)$ \\
\hline Small Uterus & $2(0.44)$ \\
\hline Endometrial Polyp & $3(0.66)$ \\
\hline \multicolumn{2}{|l|}{ HSG } \\
\hline Both Tubes Normal & $347(75.95)$ \\
\hline One Tube Blocked & $72(15.81)$ \\
\hline Both Tubes Blocked & $35(8.25)$ \\
\hline \multicolumn{2}{|l|}{ LH Category } \\
\hline$\leq 10$ & $408(90.04)$ \\
\hline$>10$ & $45(9.96)$ \\
\hline \multicolumn{2}{|l|}{ FSH Category } \\
\hline$\leq 10$ & $416(91.63)$ \\
\hline$>10$ & $38(8.37)$ \\
\hline \multicolumn{2}{|l|}{ TSH Category } \\
\hline$\leq 4.5$ & $344(76.1)$ \\
\hline$>4.5$ & $108(23.9)$ \\
\hline \multicolumn{2}{|l|}{ Prolactin } \\
\hline$\leq 25$ & $402(88.9)$ \\
\hline$>25$ & $50(11.1)$ \\
\hline \multicolumn{2}{|l|}{ HSA } \\
\hline All Parameter Normal & $283(62.61)$ \\
\hline Any one parameter abnormal & $104(23.01)$ \\
\hline Azoospermia & $65(14.38)$ \\
\hline
\end{tabular}

Table 2 presents the clinical characteristics of women. Nearly $68 \%$ women had primary infertility and rest had secondary infertility. Out of 454 women, 135 women reported irregular menstrual cycles in which 64(47\%) had oligomenorrhoea. In $79 \%$ women, pelvic ultrasonography was normal and nearly $11 \%$ of women had features of PCOD.

As far as HSG is concerned, nearly $16 \%$ women were found to have one tube blocked and $8 \%$ had both tube blocked. Nearly $10 \%$ of the women in study were found to have high level of LH i.e. more than $10 \mathrm{IU} / \mathrm{L}$. Nearly $8 \%$ of women had high level of FSH i.e. more than 10 IU/L which is an indicator of ovarian resistance.

Out of 454 women, 109 (24\%) women had high level of TSH i.e. more than $4.5 \mathrm{IU} / \mathrm{L}$ indicating high proportion of hypothyroidism among the study population. High TSH level typically indicates subclinical or clinical hypothyroidism. Out of 454 women tested for prolactin level, 59(13\%) were found to have high level of prolactin i.e. $>25 \mathrm{ng} / \mathrm{ml}$. The husband semen analysis (HSA) was found to be abnormal with at least one parameter in $23 \%$ of husbands of studied subjects. Nearly $14 \%$ of their male partners suffered from azoospermia.

\section{DISCUSSION}

Inability to produce a child is a social stigma in Northeastern part of India and it may lead to broken marriage, marital disharmony, and second marriage but very rarely to child adoption. The mean age of women at presentation was 28.24 years (95\% CI: 27.7-28.8 years).

The median duration of infertility at presentation was 5 years (IQR: 3-9 years). The delay in seeking treatment may be due to previous visits to quacks, unawareness of fertility treatment, poverty, poor access to appropriate treatment, prior unsuccessful medical treatment, depression, social stigma.

Nearly $72 \%$ of couple reported to hospital for treatment between 2-7 years of marriage. Presentation after five years of marriage was seen in $53 \%$ of patients in study of Nigerian population. ${ }^{12}$ The predominance of primary infertility $(67.55 \%)$ in our study agrees with the pervious study of most western societies where primary infertility is seen in almost (61-70\%) of cases and secondary infertility in $29-33 \%$ of patients. ${ }^{13,14}$

Present study is in contrast with Panti AA et al study done in Nigeria where a total of 1,264 new gynecological cases were seen during the study period, and 198 infertile patients were evaluated. The prevalence of infertility was $15.7 \%$. Primary infertility constituted $32.8 \%$, while secondary infertility was $67.2 \% .^{12}$

Male factor was responsible in $37.39 \%$, female factor in $20.48 \%$, unexplained in $22.46 \%$ while a combination of both factor was seen in $8.37 \%$ cases in our study while in study conducted by Chowdhary MA et al female accounted for $45.8 \%$, male factor in $25.6 \%$, unexplained 
in $9.8 \%$ and in $18.8 \%$ both partner had abnormality. ${ }^{15}$ The study done in Nigeria shows Female gender-related causes of infertility accounted for $42.9 \%$; male causes accounted for $19.7 \% .^{12}$ Both partners contributed to infertility in $16.7 \%$, while no cause was found in $20.7 \%$ of patients.

On analyzing the cause of female factor infertility, it was found that $20.48 \%$ women had uterine, ovarian or tubal pathology diagnosed by USG, $24.05 \%$ women has one or both tubes blocked on HSG, $8.37 \%$ women showed poor ovarian reserve. Tubal factor infertility was the leading cause followed by subclinical/clinical hypothyroidism $(23.9 \%)$.

In Mongolian study, tubal factor infertility was seen in $32.8 \%$ of women Female factor was responsible in $45.8 \%$ of couple, male factor was seen in $25.6 \%$ of case and in $18.8 \%$ of couples both partners had demonstrable couple of infertility while $9.8 \%$.has unexplained infertility. ${ }^{16}$

According to study by Fathi et al primary infertility accounted for $65 \%$ of cases. ${ }^{17}$ Causes of infertility were male factor $(45 \%)$, oligo-ovulation disorders $(37 \%)$ and tubal damage (18\%). Infertility factors were identified in the woman alone in $30.6 \%$ of cases and the man alone in $29.2 \%$. Two combined infertility factors were found in $18 \%$ of patients, and three combined factors in $0.5 \%$. The rate of unexplained infertility (which probably includes non-tubal endometriosis) was $20.7 \%$.

In a study conducted by Masoumi et al in Iran, the prevalence of primary and secondary infertility was $69.5 \%$ and $30.5 \%$ respectively. ${ }^{18}$

Among the various causes of infertility women factors $(88.6 \%)$ had the highest regard. In the causes of female infertility, menstrual disorders, diseases (obesity, thyroid diseases, and diabetes), ovulation dysfunction, uterine factor, fallopian tubes and cervical factor had the highest prevalence respectively. The causes of male infertility based on their frequency included semen fluid abnormalities, genetic factors, vascular abnormalities, and anti-spermatogenesis factors, respectively.

\section{CONCLUSION}

Etiological pattern of infertility varies in different parts of World. Male and female factors both are responsible for infertility. So, both the partners should be counselled and investigated properly before proceeding to aggressive infertility treatments. There was a paucity of studies related to etiological pattern of infertility. So, this study has been done to know the cause and clinical pattern of infertility in married couples in Bihar.

Funding: No funding sources Conflict of interest: None declared

Ethical approval: The study was approved by the Institutional Ethics Committee

\section{REFERENCES}

1. World Health Organization (WHO). Mother or nothing: The agony of infertility. WHO Bulletin. 2010; 88:877-953.

2. Inhorn MC. Global infertility and the globalization of new reproductive technologies: Illustrations from Egypt. Soc Sci Med. 2003;56:1837-51.

3. World Health Organisation. 1994. Challenges in reproductive health research, Biennial report 19921993, Geneva.

4. World Health Organization. Infecundity, infertility, and childlessness in developing countries. DHS Comparative Reports No 9. Calverton, Maryland, USA: ORC Macro and the World Health Organization; 2004.

5. Talwar PP, Go OP, Murali IN. Prevalence of infertility in different population groups in India and its determinants. In: Statistics and demography. New Delhi: National Institute of Health \& Family Welfare \& Indian Council of Medical Research; 1986.

6. Unisa S. Childlessness in Andhra Pradesh, India: Treatment seeking and consequences. Reprod Health Matters. 1999; 7: 54-64.

7. Zargar AH, Wani AI, Masoodi SR, Laway BA, Salahuddin M. Epidemiologic and etiologic aspects of primary infertility in the Kashmir region of India. Fertil Steril. 1997;68:637-43.

8. Kumar D. Prevalence of female infertility and its socioeconomic factors in tribal communities of Central India. Rural Remote Health. 2007;7:456.

9. Jejeebhoy SJ. Infertility in India - levels, patterns and consequences: priorities for social science research. J Family Welfare. 1998;44(2):15-24.

10. Ganguly S, Unisa S. Trends of Infertility and Childlessness in India: Findings from NFHS Data. Facts Views Vis Obgyn. 2010;2 (2):131-8.

11. Adamson PC, Krupp K, Alexandra H, Freeman AH, Klausner JD, Arthur L et al. Prevalence and correlates of primary infertility among young women in Mysore, India. Indian J Med Res. 2011;134:440-6.

12. Panti AA, Sununu YT. The profile of infertility in a teaching Hospital in North West Nigeria. Sahel Med J. 2014;17(1):7-11.

13. Okonofua FE, Odunsi K. Infertility in Sub -Saharan Africa. Contemporary obstetrics and gynaecology for developing countries. Publishers: Women's Health and Action Research Centre. Benin city. 2003.p.12856.

14. Templeton A, Fraser C, Thompson B. Infertilityepidermiology and referral practice. Hum Reprod. 1991;6:1391-4.

15. Chowdhary MA, Haque MM, Choudhary S,Prodhania MS. Determinants of Infertility Among Couples Seeking Treatment in A Selected Clinic in Dhaka City. Chattagsam Maa-O-Shishu Hospital Med College J. 2014;13(3):42-5.

16. Bayasgalan et al. Clinic pattern and major causes of infertility in Mangolia. Obstet Gynaecol Res. 2004;30(5):386-93. 
17. Masoumi SZ, Parsa P, Darvish N, Mokhtari S, Yavangi M, Roshanaei G. An epidemiologic survey on the causes of infertility in patients referred to infertility center in Fatemieh Hospital in Hamadan. Iran J Reprod Med. 2015;13(8):513-6.

18. Fathi J, Haroush Ben. Distribution of causes of infertility in patients attending primary fertility clinics in Isreal. Isreal Med Assoc J. 2011;13:51-4.
Cite this article as: Singh K, Kumari R, Ranjan A, Bharti G. Analysis of causes and clinical pattern of infertility in couples coming to a tertiary care centre in Bihar, India. Int J Reprod Contracept Obstet

Gynecol 2017;6:2279-2283. 